\title{
The 2D Dirichlet Problem for the Propagative Helmholtz Equation in an Exterior Domain with Cracks and Singularities at the Edges
}

\author{
P. A. Krutitskii \\ KIAM, Miusskaya Square 4, Moscow 125047, Russia \\ Correspondence should be addressed to P. A. Krutitskii, krutitsk@mail.ru
}

Received 30 March 2012; Accepted 3 May 2012

Academic Editor: Vladimir Mityushev

Copyright (C) 2012 P. A. Krutitskii. This is an open access article distributed under the Creative Commons Attribution License, which permits unrestricted use, distribution, and reproduction in any medium, provided the original work is properly cited.

The Dirichlet problem for the 2D Helmholtz equation in an exterior domain with cracks is studied. The compatibility conditions at the tips of the cracks are assumed. The existence of a unique classical solution is proved by potential theory. The integral representation for a solution in the form of potentials is obtained. The problem is reduced to the Fredholm equation of the second kind and of index zero, which is uniquely solvable. The asymptotic formulae describing singularities of a solution gradient at the edges (endpoints) of the cracks are presented. The weak solution to the problem may not exist, since the problem is studied under such conditions that do not ensure existence of a weak solution.

\section{Introduction}

The 2D Dirichlet boundary value problem for the Helmholtz equation in an exterior multiply connected domain bounded by closed curves is considered in monographs on mathematical physics, for instance, in [1-3]. The review on studies of the Dirichlet problem for this equation in the exterior of cracks is given in [4]. The present paper is an attempt to combine these problems and to consider exterior domains containing cracks. From a practical stand point such domains have great significance, because cracks model both cracks in solids and wings or double-sided screens in fluids.

So, we study Dirichlet problem in an exterior domain bounded by closed curves and cracks. The theorems on existence and uniqueness of a classical solution are proved. The integral representation for a solution to a problem in the form of potentials is obtained. The problem is reduced to the uniquely solvable Fredholm integral equation of the second kind and index zero. To derive uniquely solvable integral equation on the whole boundary we use 
the modified integral equation approach $[5,6]$ on the closed curves. Since the derived integral equation of the 2nd kind is uniquely solvable, we may obtain its numerical solution in a very simple way, just by discretization and inversion of the matrix. Substituting numerical solution of the integral equation into potentials, we obtain numerical solution to the exterior Dirichlet problem in a very simple way as well. The integral representation for a solution presented in the present paper enables us to derive asymptotic formulae for singularities of a gradient of the solution at the tips of the cracks.

The Dirichlet problem for the Helmholtz equation in the exterior of several closed curves in a plane and the Dirichlet problem for the Helmholtz equation in the exterior of several curvilinear cracks in a plane are particular cases of our problem.

It is important to stress that the boundary data on the closed curves in the present paper is assumed to be only continuous. This means that weak solution may not exist in our problem, though classical solution exists. In other words, the problem in this paper is studied under conditions, which are not sufficient for existence of a weak solution in $H_{\mathrm{loc}}^{1}$ and weak solution may not exist, but these conditions are sufficient for existence of a classical solution. This curious fact follows from the Hadamard example of a nonexistence of a weak solution to the Dirichlet problem for Laplacian in the unit disc with continuous boundary data (classical solution exists in this example). Roughly speaking, continuity of a Dirichlet data on smooth closed curves does not ensure existence of a weak solution in the Dirichlet problem. The Hadamard example of existence of a classical solution and nonexistence of a weak solution is presented and is discussed in the book [7, section 12.5] by Sobolev himself, who invented Sobolev's spaces for analysis of weak solvability of boundary value problems.

Numerical methods for the Dirichlet and Neumann problems for the Laplace and Helmholtz equations in the exterior of cracks in a plane have been developed in $[8,9]$ on the basis of boundary integral equations. Numerical simulation for engineering problems with cracks is presented in [10-12] using boundary element method. Problems with a crack in electromagnetoelasticity have been reduced to integral equations in [13], and numerical solutions for some model problems have been obtained. The Dirichlet problem for elasticity equations in an exterior of several arbitrary curvilinear cracks in a plane has been reduced to the uniquely solvable integral equations in [14].

\section{Formulation of the Problem}

By an open curve we mean a simple smooth nonclosed arc of finite length without selfintersections [15]. In the plane $x=\left(x_{1}, x_{2}\right) \in R^{2}$ we consider an exterior multiply connected domain bounded by simple open curves $\Gamma_{1}^{1}, \ldots, \Gamma_{N_{1}}^{1} \in C^{2, \lambda}$ and simple closed curves $\Gamma_{1}^{2}, \ldots, \Gamma_{N_{2}}^{2} \in C^{2, \lambda}, \lambda \in(0,1]$, so that the curves do not have common points; in particular, they do not have common endpoints. Suppose that $N_{1}+N_{2}>0$. We set $\Gamma^{1}=\bigcup_{n=1}^{N_{1}} \Gamma_{n}^{1}$, $\Gamma^{2}=\bigcup_{n=1}^{N_{2}} \Gamma_{n}^{2}$, and $\Gamma=\Gamma^{1} \cup \Gamma^{2}$. The exterior connected domain bounded by $\Gamma^{2}$ will be denoted by $\Phi$. We assume that each curve $\Gamma_{n}^{k}$ is parametrized by the arc length $s$ :

$$
\Gamma_{n}^{k}=\left\{x: x=x(s)=\left(x_{1}(s), x_{2}(s)\right), s \in\left[a_{n}^{k}, b_{n}^{k}\right]\right\}, \quad n=1, \ldots, N_{k}, k=1,2
$$

so that $a_{1}^{1}<b_{1}^{1}<\cdots<a_{N_{1}}^{1}<b_{N_{1}}^{1}<a_{1}^{2}<b_{1}^{2}<\cdots<a_{N_{2}}^{2}<b_{N_{2}}^{2}$ and the domain $\Phi$ is to the right when the parameter $s$ increases on $\Gamma_{n}^{2}$. Therefore points $x \in \Gamma$ and values of the parameter $s$ are in one-to-one correspondence except for $a_{n}^{2}$ and $b_{n}^{2}$, which correspond 


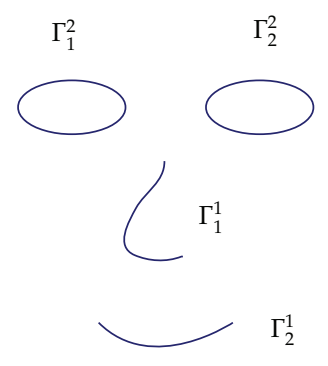

Figure 1: An example of an exterior domain.

to the same point $x$ for $n=1, \ldots, N_{2}$. Below the sets of the intervals on the $O s$ axis $\bigcup_{n=1}^{N_{1}}\left[a_{n}^{1}, b_{n}^{1}\right], \bigcup_{n=1}^{N_{2}}\left[a_{n}^{2}, b_{n}^{2}\right], \bigcup_{k=1}^{2} \bigcup_{n=1}^{N_{k}}\left[a_{n}^{k}, b_{n}^{k}\right]$ will be denoted by $\Gamma^{1}, \Gamma^{2}$, and $\Gamma$ also.

We set $C^{0}\left(\Gamma_{n}^{2}\right)=\left\{\mathcal{F}(s): \mathcal{F}(s) \in C^{0}\left[a_{n}^{2}, b_{n}^{2}\right], \mathcal{F}\left(a_{n}^{2}\right)=\mathcal{F}\left(b_{n}^{2}\right)\right\}$, and

$$
C^{0}\left(\Gamma^{2}\right)=\bigcap_{n=1}^{N_{2}} C^{0}\left(\Gamma_{n}^{2}\right)
$$

The tangent vector to $\Gamma$ at the point $x(s)$ is denoted by $\tau_{x}=(\cos \alpha(s), \sin \alpha(s))$, where $\cos \alpha(s)=x_{1}^{\prime}(s)$, and $\sin \alpha(s)=x_{2}^{\prime}(s)$. Let $\mathbf{n}_{x}=(\sin \alpha(s),-\cos \alpha(s))$ be the normal vector to $\Gamma$ at $x(s)$. The direction of $\mathbf{n}_{x}$ is chosen such that it will coincide with the direction of $\tau_{x}$ if $\mathbf{n}_{x}$ is rotated anticlockwise through an angle of $\pi / 2$.

We consider $\Gamma^{1}$ as a set of cracks. The side of $\Gamma^{1}$ which is on the left, when the parameter $s$ increases, will be denoted by $\left(\Gamma^{1}\right)^{+}$and the opposite side will be denoted by $\left(\Gamma^{1}\right)^{-}$.

We say that the function $w(x)$ belongs to the smoothness class $\mathbf{K}$ if

(1) $w \in C^{0}\left(\overline{\Phi \backslash \Gamma^{1}}\right) \cap C^{2}\left(\Phi \backslash \Gamma^{1}\right)$,

(2) $\nabla w \in C^{0}\left(\overline{\Phi \backslash \Gamma^{1}} \backslash \Gamma^{2} \backslash X\right)$, where $X$ is a point-set, consisting of the endpoints of $\Gamma^{1}: X=\bigcup_{n=1}^{N_{1}}\left(x\left(a_{n}^{1}\right) \cup x\left(b_{n}^{1}\right)\right)$,

(3) in the neighbourhood of any endpoint $x(d) \in X$ for some constants $\mathcal{C}>0, \epsilon>-1$ the inequality

$$
|\nabla w| \leq \mathcal{C}|x-x(d)|^{e}
$$

holds, where $x \rightarrow x(d)$ and $d=a_{n}^{1}$ or $d=b_{n}^{1}$ for $n=1, \ldots, N_{1}$.

Remark 2.1. By $C^{0}\left(\overline{\Phi \backslash \Gamma^{1}} \backslash \Gamma^{2} \backslash X\right)$ we denote the class of continuous in $\Phi \backslash \Gamma^{1}$ functions, which are continuously extensible to the sides of the cracks $\Gamma^{1} \backslash X$ from the left and from the right, but their limiting values on $\Gamma^{1} \backslash X$ can be different from the left and from the right, so that these functions may have a jump on $\Gamma^{1} \backslash X$. The functions of class $C^{0}\left(\bar{\Phi} \backslash \Gamma^{1} \backslash \Gamma^{2} \backslash X\right)$ belong to the class $C^{0}\left(\overline{\Phi \backslash \Gamma^{1}}\right)$ if they are continuously extensible to $\Gamma^{2}$ from $\Phi$ and if they are continuously extensible to the tips of the cracks $\Gamma^{1}$.

Let us formulate the Dirichlet problem for the Helmholtz equation in the exterior domain $\Phi \backslash \Gamma^{1}$ (see Figure 1). 
Problem $U$. Find a function $u(x)$ of the class $\mathbf{K}$ which satisfies the Helmholtz equation:

$$
u_{x_{1} x_{1}}(x)+u_{x_{2} x_{2}}(x)+\beta^{2} u(x)=0, \quad x \in \Phi \backslash \Gamma^{1}, \beta=\text { const }>0,
$$

the boundary conditions:

$$
\left.u(x(s))\right|_{\left(\Gamma^{1}\right)^{+}}=F^{+}(s),\left.\quad u(x(s))\right|_{\left(\Gamma^{1}\right)^{-}}=F^{-}(s),\left.\quad u(x(s))\right|_{\Gamma^{2}}=F(s),
$$

and the radiating conditions at infinity:

$$
u=O\left(|x|^{-1 / 2}\right), \quad \frac{\partial u}{\partial|x|}-i \beta u=o\left(|x|^{-1 / 2}\right), \quad|x|=\sqrt{x_{1}^{2}+x_{2}^{2}} \longrightarrow \infty
$$

All conditions of the Problem $U$ must be satisfied in the classical sense. Problem $U$ includes two particular cases. In the first particular case, there are no cracks $\Gamma^{1}$ (i.e., $\Gamma^{1}=\emptyset$ ), then we get the Dirichlet problem for the Helmholtz equation in the exterior of several closed curves $\Gamma^{2}$ in a plane (see [1-3]). In another particular case, there are no closed curves $\Gamma^{2}$ (i.e., $\Gamma^{2}=\emptyset$ ), and we obtain the Dirichlet problem for the Helmholtz equation in the exterior of several curvilinear cracks $\Gamma^{1}$ in a plane [4].

By $\int_{\Gamma^{k}} \cdots d \sigma$ we mean $\sum_{n=1}^{N_{k}} \int_{a_{n}^{k}}^{b_{n}^{k}} \cdots d \sigma$. On the basis of the Rellich lemma [1], energy equalities [2], and the regularity of the solution to the homogeneous Dirichlet problem near the boundary $\Gamma^{2}$ (see [16, lemma 6.18]), we can easily prove the following assertion.

Theorem 2.2. If $\Gamma \in C^{2, \lambda}, \lambda \in(0,1]$, then the Problem $U$ has at most one solution.

Proof. It is sufficiently to prove that the homogeneous Problem $U$ admits the trivial solution only. Let $u_{0}(x)$ be a solution to the homogeneous Problem $U$ with $F^{+}(s) \equiv F^{-}(s) \equiv 0$ and $F(s) \equiv 0$. Let $S_{r}$ be an open disc with a center in the origin and with sufficiently large radius $r$. Assume that $\Gamma \subset S_{r_{0}}$ for some $r_{0}$ and assume that $r>r_{0}$.

Since $\Gamma^{2} \in C^{2, \lambda}, u_{0}(x) \in C^{0}\left(\bar{\Phi} \backslash \Gamma^{1}\right)$ (remind that $u_{0}(x) \in \mathbf{K}$ ), and since $\left.u_{0}\right|_{\Gamma^{2}}=0 \in$ $C^{2, \lambda}\left(\Gamma^{2}\right)$, and owing to the lemma on regularity of solutions of elliptic equations near the boundary [16, lemma 6.18], we obtain $u_{0}(x) \in C^{1}\left(\bar{\Phi} \backslash \Gamma^{1}\right)$. Since $u_{0}(x) \in \mathbf{K}$, we observe that $u_{0}(x) \in C^{1}\left(\overline{\Phi \backslash \Gamma^{1}} \backslash X\right)$. We envelope each crack $\Gamma_{n}^{1}\left(n=1, \ldots, N_{1}\right)$ by a closed contour and write first Green's formula for $u_{0}(x)$ in a domain, bounded by these contours, by $\Gamma^{2}$ and by $\partial S_{r}$. Then we allow to shrink closed contours onto cracks $\Gamma^{1}$ and use smoothness of the function $u_{0}(x)$. In this way we arrive at the identity for $u_{0}(x)$ in the domain $\oslash \cap S_{r} \backslash \Gamma^{1}$

$$
\begin{aligned}
\left\|\nabla u_{0}\right\|_{L_{2}\left(\Phi \cap S_{r} \backslash \Gamma^{1}\right)}^{2}-\beta^{2}\left\|u_{0}\right\|_{L_{2}\left(\Phi \cap S_{r} \backslash \Gamma^{1}\right)}^{2}= & \int_{\Gamma^{1}}\left(\bar{u}_{0}\right)^{+}\left(\frac{\partial u_{0}}{\partial n_{x}}\right)^{+} d s-\int_{\Gamma^{1}}\left(\bar{u}_{0}\right)^{-}\left(\frac{\partial u_{0}}{\partial n_{x}}\right)^{-} d s \\
& -\int_{\Gamma^{2}} \bar{u}_{0} \frac{\partial u_{0}}{\partial n_{x}} d s+\int_{\partial S_{r}} \bar{u}_{0} \frac{\partial u_{0}}{\partial|x|} d l,
\end{aligned}
$$

which is true for any $r>r_{0}$, where $r_{0}$ is some constant. The curviliniear integral of the 1 st kind is taken over $\partial S_{r}$. By $\bar{u}_{0}(x)$ the complex conjugate function to $u_{0}(x)$ is denoted. Clearly, $\bar{u}_{0}(x)$ belongs to class $\mathbf{K}$ and satisfies homogeneous boundary conditions. 
By the superscripts + and - we denote the limiting values of functions on $\left(\Gamma^{1}\right)^{+}$and on $\left(\Gamma^{1}\right)^{-}$, respectively. Since $u_{0}(x)$ satisfies the homogeneous boundary condition $(2.4 \mathrm{~b})$ on $\Gamma$, we rewrite identity $(*)$ in the following form:

$$
\left\|\nabla u_{0}\right\|_{L_{2}\left(\Phi \cap S_{r} \backslash \Gamma^{1}\right)}^{2}-\beta^{2}\left\|u_{0}\right\|_{L_{2}\left(\Phi \cap S_{r} \backslash \Gamma^{1}\right)}^{2}=\int_{\partial S_{r}} \bar{u}_{0}(x) \frac{\partial u_{0}(x)}{\partial|x|} d l .
$$

Taking the imaginary part, we obtain the identity

$$
\operatorname{Im} \int_{\partial S_{r}} \bar{u}_{0}(x) \frac{\partial u_{0}(x)}{\partial|x|} d l=0
$$

which is true for any $r>r_{0}$. Tending $r \rightarrow \infty$ in this identity and taking into account conditions $(2.4 \mathrm{c})$ at infinity, we obtain

$$
\lim _{r \rightarrow \infty} \operatorname{Im} \int_{\partial S_{r}} \bar{u}_{0}(x) \frac{\partial u_{0}(x)}{\partial|x|} d l=\beta \lim _{r \rightarrow \infty} \int_{\partial S_{r}}\left|u_{0}\right|^{2} d l=0
$$

Since $\beta>0$, we have

$$
\lim _{r \rightarrow \infty} \int_{\partial S_{r}}\left|u_{0}\right|^{2} d l=0
$$

whence $u_{0}(x) \equiv 0$ in $\Phi \backslash \Gamma^{1}$ on the basis of the Rellich lemma [1, section 229]. Thus, $u_{0}(x)$ is a trivial solution to the homogeneous Problem $U$. Consequently, the homogeneous Problem $U$ has only the trivial solution, and the theorem is proved owing to the linearity of the Problem $U$.

\section{Integral Equations at the Boundary}

To prove existence of a solution to the Problem $U$, we assume that

$$
\begin{gathered}
F^{+}(s) \in C^{1, \lambda}\left(\Gamma^{1}\right), \quad F^{-}(s) \in C^{1, \lambda}\left(\Gamma^{1}\right), \quad F(s) \in C^{0}\left(\Gamma^{2}\right), \quad \lambda \in(0,1], \\
F^{+}\left(a_{n}^{1}\right)=F^{-}\left(a_{n}^{1}\right), \quad F^{+}\left(b_{n}^{1}\right)=F^{-}\left(b_{n}^{1}\right), \quad n=1, \ldots, N_{1} .
\end{gathered}
$$

The conditions (3.1b) are compatibility conditions for functions $F^{+}(s)$ and $F^{-}(s)$ at the tips of the cracks. To solve Problem $U$ we discuss some preliminary matter.

If $\boldsymbol{B}_{1}\left(\Gamma^{1}\right)$ and $\boldsymbol{B}_{2}\left(\Gamma^{2}\right)$ are Banach spaces of functions given on $\Gamma^{1}$ and $\Gamma^{2}$, then for functions given on $\Gamma$ we introduce the Banach space $\boldsymbol{B}_{1}\left(\Gamma^{1}\right) \cap \boldsymbol{B}_{2}\left(\Gamma^{2}\right)$ with the norm $\|\cdot\|_{B_{1}\left(\Gamma^{1}\right) \cap B_{2}\left(\Gamma^{2}\right)}=\|\cdot\|_{\mathcal{B}_{1}\left(\Gamma^{1}\right)}+\|\cdot\|_{B_{2}\left(\Gamma^{2}\right)}$. 
We consider the angular potential from [4] for (2.4a) on $\Gamma^{1}$ :

$$
v_{1}[v](x)=\frac{i}{4} \int_{\Gamma^{1}} v(\sigma) V(x, \sigma) d \sigma .
$$

The kernel $V(x, \sigma)$ is defined on each curve $\Gamma_{n}^{1}, n=1, \ldots, N_{1}$, by

$$
V(x, \sigma)=\int_{a_{n}^{1}}^{\sigma} \frac{\partial}{\partial \mathbf{n}_{y}} \mathscr{H}_{0}^{(1)}(\beta|x-y(\xi)|) d \xi, \quad \sigma \in\left[a_{n}^{1}, b_{n}^{1}\right]
$$

where $\mathscr{H}_{0}^{(1)}(z)$ is the Hankel function of the first kind [3]:

$$
\begin{gathered}
\mathscr{\ell}_{0}^{(1)}(z)=\frac{\sqrt{2} \exp (i z-i \pi / 4)}{\pi \sqrt{z}} \int_{0}^{\infty} \exp (-t) t^{-1 / 2}\left(1+\frac{i t}{2 z}\right)^{-1 / 2} d t \\
y=y(\xi)=\left(y_{1}(\xi), y_{2}(\xi)\right), \\
|x-y(\xi)|=\sqrt{\left(x_{1}-y_{1}(\xi)\right)^{2}+\left(x_{2}-y_{2}(\xi)\right)^{2}} .
\end{gathered}
$$

Here in after we suppose that $v(\sigma)$ belongs to $C^{0, \lambda}\left(\Gamma^{1}\right)$ and satisfies the following additional conditions:

$$
\int_{a_{n}^{1}}^{b_{n}^{1}} v(\sigma) d \sigma=0, \quad n=1, \ldots, N_{1}
$$

As shown in [4], for such $v(\sigma)$ the angular potential $v_{1}[v](x)$ belongs to the class $\mathbf{K}$. In particular, the condition (2.3) is satisfied for any $\epsilon \in(0,1)$. Moreover, integrating $v_{1}[v](x)$ by parts and using (3.5) we express the angular potential in terms of a double-layer potential:

$$
v_{1}[v](x)=-\frac{i}{4} \int_{\Gamma^{1}} \rho(\sigma) \frac{\partial}{\partial \mathbf{n}_{y}} \mathscr{H}_{0}^{(1)}(\beta|x-y(\sigma)|) d \sigma
$$

with the density

$$
\rho(\sigma)=\int_{a_{n}^{1}}^{\sigma} v(\xi) d \xi, \quad \sigma \in\left[a_{n}^{1}, b_{n}^{1}\right], n=1, \ldots, N_{1}
$$

Consequently, $v_{1}[v](x)$ satisfies both equation (2.4a) outside $\Gamma^{1}$ and the conditions at infinity (2.4c).

Let us construct a solution to the Problem $U$. This solution can be obtained with the help of potential theory for the Helmholtz equation (2.4a). We look for a solution to the problem in the following form:

$$
u[v, \mu](x)=v_{1}[v](x)+w[\mu](x),
$$


where $v_{1}[v](x)$ is given by (3.2), (3.6), and

$$
\begin{gathered}
w[\mu](x)=w_{1}[\mu](x)+w_{2}[\mu](x), \\
w_{1}[\mu](x)=\frac{i}{4} \int_{\Gamma^{1}} \mu(\sigma) \mathscr{\ell}_{0}^{(1)}(\beta|x-y(\sigma)|) d \sigma \\
w_{2}[\mu](x)=\frac{i}{4} \int_{\Gamma^{2}} \mu(\sigma)\left(\frac{\partial}{\partial \mathbf{n}_{y}}-i\right) \mathscr{\ell}_{0}^{(1)}(\beta|x-y(\sigma)|) d \sigma .
\end{gathered}
$$

The density $v(\sigma)$ must belong to $C^{0, \lambda}\left(\Gamma^{1}\right)$ and must satisfy conditions (3.5).

We will look for $\mu(s)$ in the Banach space $C_{q}^{\omega}\left(\Gamma^{1}\right) \cap C^{0}\left(\Gamma^{2}\right), \omega \in(0,1], q \in[0,1)$ with the norm $\|\cdot\|_{C_{q}^{\omega}\left(\Gamma^{1}\right) \cap C^{0}\left(\Gamma^{2}\right)}=\|\cdot\|_{C_{q}^{\omega}\left(\Gamma^{1}\right)}+\|\cdot\|_{C^{0}\left(\Gamma^{2}\right)}$. We say that $\mu(s)$ belongs to the Banach space $C_{q}^{\omega}\left(\Gamma^{1}\right)$ with some $\omega \in(0,1]$ and $q \in[0,1)$ if

$$
\mu(s) \prod_{n=1}^{N_{1}}\left|s-a_{n}^{1}\right|^{q}\left|s-b_{n}^{1}\right|^{q} \in C^{0, \omega}\left(\Gamma^{1}\right),
$$

where $C^{0, \omega}\left(\Gamma^{1}\right)$ is a Hölder space with the exponent $\omega$. The norm in the Banach space $C_{q}^{\omega}\left(\Gamma^{1}\right)$ is defined by

$$
\|\mu(\cdot)\|_{C_{q}^{\omega}\left(\Gamma^{1}\right)}=\left\|\mu(\cdot) \prod_{n=1}^{N_{1}}\left|\cdot-a_{n}^{1}\right|^{q}\left|\cdot-b_{n}^{1}\right|^{q}\right\|_{C^{0, \omega}\left(\Gamma^{1}\right)} .
$$

It can be checked directly with the help of [4] that for such $\mu(s)$ the function $w_{1}[\mu](x)$ satisfies (2.4a) and belongs to the class $\mathbf{K}$. In particular, the inequality (2.3) holds with $\epsilon=-q$ if $q \in(0,1)$. The potential $w_{2}[\mu](x)$ satisfies $(2.4 \mathrm{a})$ and belongs to $C^{0}(\bar{\Phi}) \cap C^{2}(\boldsymbol{\Phi})$. It is clear that the function (3.8) satisfies conditions at infinity (2.4c). So, the function (3.8) with densities $\mu(s)$ and $v(s)$ subject to requirements described before satisfies all conditions of the Problem $U$ except for the boundary conditions $(2.4 b)$.

To satisfy the boundary conditions we substitute (3.8) in (2.4b) and arrive at the system of integral equations for the densities $\mu(s)$ and $v(s)$ :

$$
\begin{aligned}
\pm \frac{1}{2} \rho(s) & +\frac{i}{4} \int_{\Gamma^{1}} v(\sigma) V(x(s), \sigma) d \sigma+\frac{i}{4} \int_{\Gamma^{1}} \mu(\sigma) \mathscr{H}_{0}^{(1)}(\beta|x(s)-y(\sigma)|) d \sigma \\
& +\frac{i}{4} \int_{\Gamma^{2}} \mu(\sigma)\left(\frac{\partial}{\partial \mathbf{n}_{y}}-i\right) \mathscr{L}_{0}^{(1)}(\beta|x(s)-y(\sigma)|) d \sigma=F^{ \pm}(s), \quad s \in \Gamma^{1},
\end{aligned}
$$




$$
\begin{aligned}
\frac{i}{4} \int_{\Gamma^{1}} v(\sigma) V(x(s), \sigma) d \sigma & +\frac{i}{4} \int_{\Gamma^{1}} \mu(\sigma) \mathscr{H}_{0}^{(1)}(\beta|x(s)-y(\sigma)|) d \sigma+\frac{1}{2} \mu(s) \\
& +\frac{i}{4} \int_{\Gamma^{2}} \mu(\sigma)\left(\frac{\partial}{\partial \mathbf{n}_{y}}-i\right) \mathscr{H}_{0}^{(1)}(\beta|x(s)-y(\sigma)|) d \sigma=F(s), \quad s \in \Gamma^{2},
\end{aligned}
$$

where $\rho(s)$ is defined in terms of $v(s)$ in (3.7).

To derive limit formulas for the angular potential, we used its expression in the form of a double-layer potential (3.6).

Equation (3.12a) is obtained as $x \rightarrow x(s) \in\left(\Gamma^{1}\right)^{ \pm}$and comprises two integral equations. The upper sign denotes the integral equation on $\left(\Gamma^{1}\right)^{+}$, and the lower sign denotes the integral equation on $\left(\Gamma^{1}\right)^{-}$.

In addition to the integral equations written before we have the conditions (3.5).

Subtracting the integral equations (3.12a) and using (3.7), we find

$$
\begin{gathered}
\rho(s)=\left(F^{+}(s)-F^{-}(s)\right) \in C^{1, \lambda}\left(\Gamma^{1}\right), \\
v(s)=\left(F^{\prime+}(s)-F^{\prime}-(s)\right) \in C^{0, \lambda}\left(\Gamma^{1}\right), \quad F^{\prime \pm}(s)=\frac{d}{d s} F^{ \pm}(s) .
\end{gathered}
$$

We note that $v(s)$ is found completely and satisfies all required conditions, in particular, conditions (3.5). Hence, the angular potential of (3.2) and (3.6) is found completely as well.

We introduce the function $f(s)$ on $\Gamma$ by

$$
f(s)=F(s)-\frac{i}{4} \int_{\Gamma^{1}}\left(F^{\prime}(\sigma)-F^{\prime}(\sigma)\right) V(x(s), \sigma) d \sigma, \quad s \in \Gamma,
$$

where $F(s)$ is specified on $\Gamma^{2}$ in $(2.4 \mathrm{~b})$ and $F(s)=(1 / 2)\left(F^{+}(s)+F^{-}(s)\right)$ if $s \in \Gamma^{1}$. As shown in [4], if $s \in \Gamma^{1}$, then $f(s) \in C^{1, p_{0}}\left(\Gamma^{1}\right)$ where $p_{0}=\lambda$ if $0<\lambda<1$ and $p_{0}=1-\epsilon_{0}$ for any $\epsilon_{0} \in(0,1)$ if $\lambda=1$. Consequently, $f(s) \in C^{1, p_{0}}\left(\Gamma^{1}\right) \cap C^{0}\left(\Gamma^{2}\right)$.

We set

$$
\delta(s)= \begin{cases}0 & \text { if } s \in \Gamma^{1}, \\ 1 & \text { if } s \in \Gamma^{2} .\end{cases}
$$

Adding the integral equations (3.12a) and taking into account (3.12b) we obtain the integral equation for $\mu(s)$ on $\Gamma$ :

$$
\begin{aligned}
\frac{i}{4} \int_{\Gamma^{1}} & \mu(\sigma) \mathscr{\ell}_{0}^{(1)}(\beta|x(s)-y(\sigma)|) d \sigma+\frac{1}{2} \delta(s) \mu(s) \\
& +\frac{i}{4} \int_{\Gamma^{2}} \mu(\sigma)\left(\frac{\partial}{\partial \mathbf{n}_{y}}-i\right) \mathscr{H}_{0}^{(1)}(\beta|x(s)-y(\sigma)|) d \sigma=f(s), \quad s \in \Gamma,
\end{aligned}
$$

where $f(s)$ is given in (3.14). 
Thus, if $\mu(s)$ is a solution of (3.16) in the space $C_{q}^{\omega}\left(\Gamma^{1}\right) \cap C^{0}\left(\Gamma^{2}\right), \omega \in(0,1], q \in[0,1)$, then the potential (3.8) with $v(s)$ from (3.13) satisfies all conditions of the Problem $U$. We arrive at the following statement.

Theorem 3.1. If $\Gamma \in C^{2, \lambda}$, if conditions (3.1a) and (3.1b) hold, and if equation (3.16) has a solution $\mu(s)$ from the Banach space $C_{q}^{\omega}\left(\Gamma^{1}\right) \cap C^{0}\left(\Gamma^{2}\right), \omega \in(0,1], q \in[0,1)$, then a solution to the Problem $U$ exists and is given by formula (3.8), where $v(s)$ is defined in (3.13).

If $s \in \Gamma^{2}$, then (3.16) is an equation of the second kind. If $s \in \Gamma^{1}$, then (3.16) is an equation of the first kind and its kernel has a logarithmic singularity, because

$$
\mathscr{H}_{0}^{(1)}(z)=\frac{2 i}{\pi} \ln \frac{z}{\beta}+h(z)
$$

where $h(z)$ is a smooth function. Indeed, as $z \rightarrow 0+0$,

$$
h(z)=\text { const }+O\left(z^{2} \ln z\right), \quad h^{\prime}(z)=O(z \ln z), \quad h^{\prime \prime}(z)=O(\ln z) .
$$

Our further treatment will be aimed to the proof of the solvability of equation (3.16) in the Banach space $C_{q}^{\omega}\left(\Gamma^{1}\right) \cap C^{0}\left(\Gamma^{2}\right)$. Moreover, we reduce equation (3.16) to a Fredholm equation of the second kind and of index zero, which can be easily computed by classical methods.

By differentiating equation (3.16) on $\Gamma^{1}$ we reduce it to the following singular integral equation on $\Gamma^{1}$ :

$$
\begin{aligned}
\frac{\partial}{\partial s} w[\mu](x(s))= & \frac{1}{2 \pi} \int_{\Gamma^{1}} \mu(\sigma) \frac{\sin \varphi_{0}(x(s), y(\sigma))}{|x(s)-y(\sigma)|} d \sigma \\
& +\frac{i}{4} \int_{\Gamma^{1}} \mu(\sigma) \frac{\partial}{\partial s} h(\beta|x(s)-y(\sigma)|) d \sigma \\
& +\frac{i}{4} \int_{\Gamma^{2}} \mu(\sigma)\left(\frac{\partial}{\partial \mathbf{n}_{y}}-i\right) \frac{\partial}{\partial s} \mathcal{H}_{0}^{(1)}(\beta|x(s)-y(\sigma)|) d \sigma=f^{\prime}(s), \quad s \in \Gamma^{1},
\end{aligned}
$$

where the function $h(z)$ is defined by $(3.17)$, and $\varphi_{0}(x, y)$ is the angle between the vector $\overrightarrow{x y}$ and the direction of the normal $\mathbf{n}_{x}$. The angle $\varphi_{0}(x, y)$ is taken to be positive if it is measured anticlockwise from $n_{x}$ and negative if it is measured clockwise from $\mathbf{n}_{x}$. Besides, $\varphi_{0}(x, y)$ is continuous in $x, y \in \Gamma$ if $x \neq y$.

Equation (3.16) on $\Gamma^{2}$ we rewrite in the following form:

$$
\mu(s)+\int_{\Gamma} \mu(\sigma) A_{2}(s, \sigma) d \sigma=2 f(s), \quad s \in \Gamma^{2},
$$


where

$$
\begin{aligned}
A_{2}(s, \sigma) & =\left\{\frac{i}{2}(1-\delta(\sigma)) \mathscr{\ell}_{0}^{(1)}(\beta|x(s)-y(\sigma)|)+\frac{i}{2} \delta(\sigma)\left(\frac{\partial}{\partial \mathbf{n}_{y}}-i\right) \mathscr{\ell}_{0}^{(1)}(\beta|x(s)-y(\sigma)|)\right\} \\
& =\frac{i}{\pi} \delta(\sigma) \ln |x(s)-y(\sigma)|+I_{1}(x(s), y(\sigma)) \\
& =\frac{i}{\pi} \delta(\sigma) \frac{I_{2}(x(s), y(\sigma))}{|x(s)-y(\sigma)|^{1 / 3}}+I_{1}(x(s), y(\sigma)) .
\end{aligned}
$$

Here $I_{1}(x(s), y(\sigma)) \in C^{0}\left(\Gamma^{2} \times \Gamma\right)$ (see [3, page 339]),

$$
I_{2}(x(s), y(\sigma))=|x(s)-y(\sigma)|^{1 / 3} \ln |x(s)-y(\sigma)| \in C^{0}\left(\Gamma^{2} \times \Gamma^{2}\right) .
$$

We note that (3.19a) is equivalent to (3.16) on $\Gamma^{1}$ if and only if (3.19a) is accompanied by the following additional conditions:

$$
w[\mu]\left(x\left(a_{n}^{1}\right)\right)=f\left(a_{n}^{1}\right), \quad n=1, \ldots, N_{1} .
$$

The system of (3.19a), (3.19b), and (3.22) is equivalent to (3.16).

It can be easily proved (see [4] for details) that

$$
\left(\frac{\sin \varphi_{0}(x(s), y(\sigma))}{|x(s)-y(\sigma)|}-\frac{1}{\sigma-s}\right) \in C^{0, \lambda}\left(\Gamma^{1} \times \Gamma^{1}\right) .
$$

Therefore we can rewrite (3.19a) in the following form:

$$
2 \frac{\partial}{\partial s} w[\mu](x(s))=\frac{1}{\pi} \int_{\Gamma^{1}} \mu(\sigma) \frac{d \sigma}{\sigma-s}+\int_{\Gamma} \mu(\sigma) Y(s, \sigma) d \sigma=2 f^{\prime}(s), \quad s \in \Gamma^{1},
$$

where (see [4])

$$
\begin{aligned}
Y(s, \sigma)= & (1-\delta(\sigma))\left[\frac{1}{\pi}\left(\frac{\sin \varphi_{0}(x(s), y(\sigma))}{|x(s)-y(\sigma)|}-\frac{1}{\sigma-s}\right)+\frac{i}{2} \frac{\partial}{\partial s} h(\beta|x(s)-y(\sigma)|)\right] \\
& \left.+\frac{i}{2} \delta(\sigma)\left(\frac{\partial}{\partial \mathbf{n}_{y}}-i\right) \frac{\partial}{\partial s} \mathcal{H}_{0}^{(1)}(\beta|x(s)-y(\sigma)|)\right\} \in C^{0, p_{0}}\left(\Gamma^{1} \times \Gamma\right), \\
& p_{0}=\lambda \text { if } 0<\lambda<1, \text { and } p_{0}=1-\epsilon_{0} \text { for any } \epsilon_{0} \in(0,1) \text { if } \lambda=1 .
\end{aligned}
$$


International Journal of Mathematics and Mathematical Sciences

\section{The Fredholm Integral Equation and the Solution to the Problem}

Inverting the singular integral operator in (3.24), we arrive at the following integral equation of the second kind $[4,15]$ :

$$
\mu(s)+\frac{1}{Q_{1}(s)} \int_{\Gamma} \mu(\sigma) A_{1}(s, \sigma) d \sigma+\frac{1}{Q_{1}(s)} \sum_{n=0}^{N_{1}-1} G_{n} s^{n}=\frac{1}{Q_{1}(s)} \Phi_{1}(s), \quad s \in \Gamma^{1},
$$

where

$$
\begin{aligned}
& A_{1}(s, \sigma)=-\frac{1}{\pi} \int_{\Gamma^{1}} \frac{Y(\xi, \sigma)}{\xi-s} Q_{1}(\xi) d \xi, \\
& Q_{1}(s)=\prod_{n=1}^{N_{1}}\left|\sqrt{s-a_{n}^{1}} \sqrt{b_{n}^{1}-s}\right| \operatorname{sign}\left(s-a_{n}^{1}\right), \\
& \Phi_{1}(s)=-\frac{1}{\pi} \int_{\Gamma^{1}} \frac{2 Q_{1}(\sigma) f^{\prime}(\sigma)}{\sigma-s} d \sigma,
\end{aligned}
$$

and $G_{0}, \ldots, G_{N_{1}-1}$ are arbitrary constants. We set $\operatorname{sign}\left(s-a_{n}^{1}\right)=1$ as $s=a_{n}^{1}$; then the function $\operatorname{sign}\left(s-a_{n}^{1}\right)$ belongs to $C^{\infty}\left(\Gamma^{1}\right)$ in $s$ variable for $n=1, \ldots, N_{1}$.

It can be shown using the properties of singular integrals [15] that $\Phi_{1}(s)$ and $A_{1}(s, \sigma)$ are Hölder continuous functions if $s \in \Gamma^{1}$ and $\sigma \in \Gamma$. Consequently, any solution of (4.1) belongs to $C_{1 / 2}^{\omega}\left(\Gamma^{1}\right)$ with some $\omega \in(0,1]$, and here in after we look for $\mu(s)$ on $\Gamma^{1}$ in this space.

We set

$$
Q(s)=(1-\delta(s)) Q_{1}(s)+\delta(s), \quad s \in \Gamma .
$$

Instead of $\mu(s) \in C_{1 / 2}^{\omega}\left(\Gamma^{1}\right) \cap C^{0}\left(\Gamma^{2}\right)$ we introduce the new unknown function $\mu_{*}(s)=\mu(s) Q(s) \in C^{0, \omega}\left(\Gamma^{1}\right) \cap C^{0}\left(\Gamma^{2}\right)$ and rewrite (4.1) and (3.19b) in the form of one equation:

$$
\mu_{*}(s)+\int_{\Gamma} \mu_{*}(\sigma) Q^{-1}(\sigma) A(s, \sigma) d \sigma+(1-\delta(s)) \sum_{n=0}^{N_{1}-1} G_{n} s^{n}=\Phi(s), \quad s \in \Gamma,
$$

where

$$
A(s, \sigma)=(1-\delta(s)) A_{1}(s, \sigma)+\delta(s) A_{2}(s, \sigma), \quad \Phi(s)=(1-\delta(s)) \Phi_{1}(s)+2 \delta(s) f(s) .
$$

To derive equations for $G_{0}, \ldots, G_{N_{1}-1}$ we substitute $\mu(s)$ from (4.1) and (3.19b) in the conditions (3.22); then in terms of $\mu_{*}(s)$ we obtain

$$
\int_{\Gamma} Q^{-1}(\xi) \mu_{*}(\xi) l_{n}(\xi) d \xi+\sum_{m=0}^{N_{1}-1} B_{n m} G_{m}=H_{n}, \quad n=1, \ldots, N_{1}
$$


where

$$
\begin{gathered}
l_{n}(\xi)=-w\left[Q^{-1}(\cdot) A(\cdot, \xi)\right]\left(a_{n}^{1}\right), \quad H_{n}=-w\left[Q^{-1}(\cdot) \Phi(\cdot)\right]\left(a_{n}^{1}\right)+f\left(a_{n}^{1}\right), \\
B_{n m}=-w\left[Q^{-1}(\cdot)(1-\delta(\cdot))(\cdot)^{m}\right]\left(a_{n}^{1}\right) .
\end{gathered}
$$

By - we denote the variable of integration in the potential $w[\mu](x)$ in (3.9).

Thus, the system of (3.19a), (3.19b), and (3.22) for $\mu(s)$ has been reduced to the system of (4.4) and (4.6) for the function $\mu_{*}(s)$ and constants $G_{0}, \ldots, G_{N_{1}-1}$. It is clear from our consideration that any solution of system of (4.4) and (4.6) generates a solution to the system of (3.19a), (3.19b), and (3.22).

As noted before, $\Phi_{1}(s)$ and $A_{1}(s, \sigma)$ are Hölder continuous functions if $s \in \Gamma^{1}$, and $\sigma \in \Gamma$. More precisely (see [4]), $\Phi_{1}(s) \in C^{0, p}\left(\Gamma^{1}\right), p=\min \{1 / 2, \lambda\}$, and $A_{1}(s, \sigma)$ belongs to $C^{0, p}\left(\Gamma^{1}\right)$ in $s$ uniformly with respect to $\sigma \in \Gamma$. Using these properties we can prove the following.

Lemma 4.1. If $\Gamma \in C^{2, \lambda}, \lambda \in(0,1], \Phi(s) \in C^{0, p}\left(\Gamma^{1}\right) \cap C^{0}\left(\Gamma^{2}\right), p=\min \{\lambda, 1 / 2\}$, and if $\mu_{*}(s)$ from $C^{0}(\Gamma)$ satisfies equation (4.4), then $\mu_{*}(s) \in C^{0, p}\left(\Gamma^{1}\right) \cap C^{0}\left(\Gamma^{2}\right)$.

The condition $\Phi(s) \in C^{0, p}\left(\Gamma^{1}\right) \cap C^{0}\left(\Gamma^{2}\right)$ holds if conditions (3.1a) and (3.1b) hold.

Hence here in after we will look for $\mu_{*}(s)$ from $C^{0}(\Gamma)$.

Since $A_{1}(s, \sigma) \in C^{0}\left(\Gamma^{1} \times \Gamma\right)$, and due to the special representation for $A_{2}(s, \sigma)$ from (3.19b), the integral operator from (4.4)

$$
\mathbf{A} \mu_{*}=\int_{\Gamma} \mu_{*}(\sigma) Q^{-1}(\sigma) A(s, \sigma) d \sigma= \begin{cases}\int_{\Gamma} \mu_{*}(\sigma) Q^{-1}(\sigma) A_{1}(s, \sigma) d \sigma & \text { if } s \in \Gamma^{1}, \\ \int_{\Gamma} \mu_{*}(\sigma) Q^{-1}(\sigma) A_{2}(s, \sigma) d \sigma & \text { if } s \in \Gamma^{2},\end{cases}
$$

is a compact operator mapping $C^{0}(\Gamma)$ into itself. Indeed, one can check using Arzela theorem [17] that the integral operator with the kernel $A_{1}(s, \sigma)$ is a compact operator mapping $C^{0}(\Gamma)$ into $C^{0}\left(\Gamma^{1}\right)$, while the integral operator with the kernel $A_{2}(s, \sigma)$ is a compact operator mapping $C^{0}\left(\Gamma^{1}\right)$ into $C^{0}\left(\Gamma^{2}\right)$. Moreover, it can be verified directly with the help of the Arzela theorem that the integral operator $\int_{\Gamma} \mu_{*}(\sigma) Q^{-1}(\sigma) A_{2}(s, \sigma) d \sigma$ is a compact operator mapping $C^{0}\left(\Gamma^{2}\right)$ into $C^{0}\left(\Gamma^{2}\right)$. To verify equicontinuity in the Arzela theorem, we may use the property of uniform continuity in $x$ on $\Gamma^{2}$ for the functions $|x-y|^{1 / 3}$ and $|x-y|^{1 / 3} \ln |x-y|$. In doing so, we may use the Cauchy-Bunyakovski inequality for estimates.

We rewrite (4.4) in the following operator form:

$$
(I+\mathbf{A}) \mu_{*}+P G=\Phi
$$

where $P$ is the operator of multiplication of the row $P=(1-\delta(s))\left(s^{0}, \ldots, s^{N_{1}-1}\right)$ by the column $G=\left(G_{0}, \ldots, G_{N_{1}-1}\right)^{T}$. The operator $P$ is finite-dimensional from $E_{N_{1}}$ into $C^{0}(\Gamma)$ and therefore compact [18]. 
Now we rewrite (4.6) in the following form:

$$
I_{N_{1}} G+L \mu_{*}+\left(B-I_{N_{1}}\right) G=H,
$$

where $H=\left(H_{1}, \ldots, H_{N_{1}}\right)^{T}$ is a column of $N_{1}$ elements, $I_{N_{1}}$ is the identity operator in $E_{N_{1}}$, $B$ is a $N_{1} \times N_{1}$ matrix consisting of the elements $B_{n m}$ from (4.7). The operator $L$ acts from $C^{0}(\Gamma)$ into $E_{N_{1}}$, so that $L \mu_{*}=\left(L_{1} \mu_{*}, \ldots, L_{N_{1}} \mu_{*}\right)^{T}$, where

$$
L_{n} \mu_{*}=\int_{\Gamma} Q^{-1}(\xi) \mu_{*}(\xi) l_{n}(\xi) d \xi
$$

The operators $\left(B-I_{N_{1}}\right)$ and $L$ are finite-dimensional and therefore compact [19].

We consider the columns

$$
\bar{\mu}=\left(\begin{array}{c}
\mu_{*} \\
G
\end{array}\right), \quad \bar{\Phi}=\left(\begin{array}{c}
\Phi \\
H
\end{array}\right)
$$

in the Banach space $C^{0}(\Gamma) \times E_{N_{1}}$ with the norm $\|\bar{\mu}\|_{C^{0}(\Gamma) \times E_{N_{1}}}=\left\|\mu_{*}\right\|_{C^{0}(\Gamma)}+\|G\|_{E_{N_{1}}}$.

We write the system of (4.9) and (4.10) in the form of one equation:

$$
(\mathbf{I}+\mathbf{R}) \bar{\mu}=\bar{\Phi}, \quad \mathbf{R}=\left(\begin{array}{cc}
\mathbf{A} & P \\
L & B-I_{N_{1}}
\end{array}\right)
$$

where $\mathbf{I}$ is the identity operator in the space $C^{0}(\Gamma) \times E_{N_{1}}$. It is clear that $\mathbf{R}$ is a compact operator mapping $C^{0}(\Gamma) \times E_{N_{1}}$ into itself, since it consists of compact operators. Therefore, (4.13) is a Fredholm equation of the second kind and of index zero in the space $C^{0}(\Gamma) \times E_{N_{1}}$ (see $[17,18,20])$.

Let us show that homogeneous equation (4.13) has only a trivial solution. Then, according to Fredholm's alternative [17, 18, 20], the inhomogeneous equation (4.13) has a unique solution for any right-hand side. Let

$$
\bar{\mu}^{0}=\left(\begin{array}{c}
\mu_{*}^{0} \\
G^{0}
\end{array}\right) \in C^{0}(\Gamma) \times E_{N_{1}}
$$

be an arbitrary solution of the homogeneous equation (4.13). According to Lemma 4.1,

$$
\bar{\mu}^{0}=\left(\begin{array}{c}
\mu_{*}^{0} \\
G^{0}
\end{array}\right) \in C^{0, p}\left(\Gamma^{1}\right) \cap C^{0}\left(\Gamma^{2}\right) \times E_{N_{1}}, \quad p=\min \left\{\lambda, \frac{1}{2}\right\} .
$$

Therefore the function $\mu^{0}(s)=\mu_{*}^{0}(s) Q^{-1}(s) \in C_{1 / 2}^{p}\left(\Gamma^{1}\right) \cap C^{0}\left(\Gamma^{2}\right)$ and the column $G^{0}$ convert the homogeneous equations (4.1), (3.19b) and (4.6) into identities. For instance, (3.19b) takes the following form:

$$
\lim _{x \rightarrow x(s) \in \Gamma^{2}} w\left[\mu^{0}\right](x)=0, \quad x \in \boldsymbol{D} .
$$


Using the homogeneous identities (4.1) and (3.19b), we check that the homogeneous identities (4.6) are equivalent to

$$
w\left[\mu^{0}\right]\left(a_{n}^{1}\right)=0, \quad n=1, \ldots, N_{1} .
$$

Besides, acting on the homogeneous identity (4.1) with a singular operator with the kernel $(s-t)^{-1}$ we observe that $\mu^{0}(s)$ satisfies the homogeneous equation (3.24):

$$
\left.\frac{\partial}{\partial s} w\left[\mu^{0}\right](x(s))\right|_{\Gamma^{1}}=0
$$

It follows from (4.16a), (4.16b) and (4.16c) that $\mu^{0}(s)$ satisfies the homogeneous equation (3.16). On the basis of Theorem 3.1, $u\left[0, \mu^{0}\right](x) \equiv w\left[\mu^{0}\right](x)$ is a solution to the homogeneous Problem $U$. According to Theorem 2.2, $w\left[\mu^{0}\right](x) \equiv 0$ for $x \in \mathscr{D} \backslash \Gamma^{1}$. Using the limit formulas for normal derivatives of a single-layer potential on $\Gamma^{1}$, we have

$$
\lim _{x \rightarrow x(s) \in\left(\Gamma^{1}\right)^{+}} \frac{\partial}{\partial \mathbf{n}_{x}} w\left[\mu^{0}\right](x)-\lim _{x \rightarrow x(s) \in\left(\Gamma^{1}\right)^{-}} \frac{\partial}{\partial \mathbf{n}_{x}} w\left[\mu^{0}\right](x)=\mu^{0}(s) \equiv 0, \quad s \in \Gamma^{1} .
$$

Hence, $w\left[\mu^{0}\right](x)=w_{2}\left[\mu^{0}\right](x) \equiv 0$ for $x \in \mathcal{D}$, and $\mu^{0}(s)$ satisfies (4.16a), which can be written as

$$
\frac{1}{2} \mu^{0}(s)+\frac{i}{4} \int_{\Gamma^{2}} \mu^{0}(\sigma)\left(\frac{\partial}{\partial \mathbf{n}_{y}}-i\right) \mathscr{\ell}_{0}^{(1)}(\beta|x(s)-y(\sigma)|) d \sigma=0, \quad s \in \Gamma^{2}
$$

It is shown in [5, page 502-504] and [6, page 187-189] that $\mu^{0}(s) \equiv 0$ is the unique solution of (4.18) in $C^{0}\left(\Gamma^{2}\right)$.

Consequently, if $s \in \Gamma$, then $\mu^{0}(s) \equiv 0$ and $\mu_{*}^{0}(s)=\mu^{0}(s) Q^{-1}(s) \equiv 0$. It follows from the homogeneous identity $(4.1)$ for $\mu^{0}(s)$ and $G_{0}^{0}, \ldots, G_{N_{1}-1}^{0}$ that $G^{0}=\left(G_{0}^{0}, \ldots, G_{N_{1}-1}^{0}\right)^{T} \equiv 0$. Hence, $\bar{\mu}^{0} \equiv 0$. Thus, the homogeneous Fredholm equation (4.13) has only a trivial solution in $C^{0}(\Gamma) \times E_{N_{1}}$.

We have proved the following assertion.

Theorem 4.2. If $\Gamma \in C^{2, \lambda}, \lambda \in(0,1]$, then (4.13) is a Fredholm equation of the second kind and of index zero in the space $C^{0}(\Gamma) \times E_{N_{1}}$. Moreover, (4.13) has a unique solution

$$
\bar{\mu}=\left(\begin{array}{c}
\mu_{*} \\
G
\end{array}\right) \in C^{0}(\Gamma) \times E_{N_{1}}
$$

for any

$$
\bar{\Phi}=\left(\begin{array}{c}
\Phi \\
H
\end{array}\right) \in C^{0}(\Gamma) \times E_{N_{1}}
$$

As a consequence of Theorem 4.2 and the Lemma 4.1 we obtain the following corollary. 
Corollary 4.3. If $\Gamma \in C^{2, \lambda}, \lambda \in(0,1]$, then equation (4.13) has a unique solution:

$$
\bar{\mu}=\left(\begin{array}{c}
\mu_{*} \\
G
\end{array}\right) \in C^{0, p}\left(\Gamma^{1}\right) \cap C^{0}\left(\Gamma^{2}\right) \times E_{N_{1}}
$$

for any

$$
\bar{\Phi}=\left(\begin{array}{l}
\Phi \\
H
\end{array}\right) \in C^{0, p}\left(\Gamma^{1}\right) \cap C^{0}\left(\Gamma^{2}\right) \times E_{N_{1}},
$$

where $p=\min \{\lambda, 1 / 2\}$.

We recall that $\bar{\Phi}$ belongs to the class of smoothness required in Corollary 4.3 if conditions (3.1a) and (3.1b) hold. Besides, (4.13) is equivalent to the system of (4.4) and (4.6). As mentioned before, if $\left\{\mu_{*}(s), G_{0}, \ldots, G_{N_{1}-1}\right\}$ is a solution of the system of (4.4) and (4.6), and $\mu_{*}(s) \in C^{0, p}\left(\Gamma^{1}\right) \cap C^{0}\left(\Gamma^{2}\right)$, then the function $\mu(s)=\mu_{*}(s) Q^{-1}(s) \in C_{1 / 2}^{p}\left(\Gamma^{1}\right) \cap C^{0}\left(\Gamma^{2}\right)$ is a solution of the system of (3.19a), (3.19b) and (3.22), and therefore $\mu(s)$ satisfies (3.16). We obtain the following statement.

Theorem 4.4. If $\Gamma \in C^{2, \lambda}$, and if conditions (3.1a) and (3.1b) hold, then equation (3.16) has a solution $\mu(s) \in C_{1 / 2}^{p}\left(\Gamma^{1}\right) \cap C^{0}\left(\Gamma^{2}\right), p=\min \{1 / 2, \lambda\}$. This solution is expressed by the formula $\mu(s)=\mu_{*}(s) Q^{-1}(s)$, where the function $\mu_{*}(s) \in C^{0, p}\left(\Gamma^{1}\right) \cap C^{0}\left(\Gamma^{2}\right)$ is found by solving the Fredholm equation (4.13), which is uniquely solvable according to Corollary 4.3.

On the basis of Theorem 3.1 we arrive at the solvability theorem for the Problem $U$.

Theorem 4.5. If $\Gamma \in C^{2, \lambda}$, and if conditions (3.1a) and (3.1b) hold, then the solution to the Problem $U$ exists and is given by (3.8), where $v(s)$ is defined in (3.13) and $\mu(s)$ is a solution of equation (3.16) in $C_{1 / 2}^{p}\left(\Gamma^{1}\right) \cap C^{0}\left(\Gamma^{2}\right), p=\min \{1 / 2, \lambda\}$ ensured by Theorem 4.4 .

It can be checked directly that the solution to the Problem $U$ satisfies condition (2.3) with $\epsilon=-1 / 2$. Explicit expressions for singularities of the solution gradient at the endpoints of the cracks will be presented in the next section.

Theorem 4.5 ensures existence of a classical solution to the Problem $U$ when $\Gamma \in C^{2, \lambda}$, $\lambda \in(0,1]$, and conditions (3.1a) and (3.1b) hold. The uniqueness of the classical solution follows from Theorem 2.2. On the basis of our consideration we suggest the following scheme for solving the Problem $U$. First, we find the unique solution of the Fredholm equation (4.13) from $C^{0}(\Gamma) \times E_{N_{1}}$. This solution automatically belongs to $C^{0, p}\left(\Gamma^{1}\right) \cap C^{0}\left(\Gamma^{2}\right) \times E_{N_{1}}$, $p=\min \{\lambda, 1 / 2\}$. Second, we construct the solution of (3.16) from $C_{1 / 2}^{p}\left(\Gamma^{1}\right) \cap C^{0}\left(\Gamma^{2}\right)$ by the formula $\mu(s)=\mu_{*}(s) Q^{-1}(s)$. Finnaly, substituting $v(s)$ from (3.13) and $\mu(s)$ in (3.8) we obtain the solution to the Problem $U$.

Remark 4.6. It is important to stress that the solution $u(x)$ to the Problem $U$, ensured by Theorem 4.5, is a classical solution, but it may be not a weak solution to the Problem $U$. In other words, classical solution to the Problem $U$ exists and is ensured by Theorem 4.5, but weak solution to the Problem $U$ may not exist in $H_{\text {loc }}^{1}\left(\boldsymbol{\Phi} \backslash \Gamma^{1}\right)$ space. This follows from the fact that Dirichlet data on the closed curves $\Gamma^{2}$ is assumed to be continuous only. Continuity of a Dirichlet boundary data on closed curves is not sufficient for existence of a weak solution 
in $H_{\mathrm{loc}}^{1}\left(\boldsymbol{\Phi} \backslash \Gamma^{1}\right)$ space. The Hadamard example of a nonexistence of a weak solution to a harmonic Dirichlet problem in a disc with continuous boundary data is given in the book [7, section 12.5] by Sobolev himself (the classical solution exists in this example).

\section{Singularities of the Gradient of the Solution at the Endpoints of the Cracks}

As noted at the end of Section 4, the gradient of the solution to Problem $U$ can be unbounded at the endpoints of the cracks $\Gamma^{1}$, so that the gradient of the solution to the Problem $U$ satisfies estimate (2.3) with the exponent $\epsilon=-1 / 2$. We will now make a detailed analysis of the behaviour of $\nabla u(x)$ at the endpoints of $\Gamma^{1}$.

Let $u(x)$ be a solution to the Problem $U$ ensured by Theorem 4.5 and given by (3.8). Let $x(d) \in X$ be one of the endpoints of $\Gamma^{1}$. In the neighbourhood of $x(d)$, we introduce the system of polar coordinates:

$$
x_{1}=x_{1}(d)+|x-x(d)| \cos \varphi, \quad x_{2}=x_{2}(d)+|x-x(d)| \sin \varphi .
$$

We will assume that $\varphi \in(\alpha(d), \alpha(d)+2 \pi)$ if $d=a_{n}^{1}$ and $\varphi \in(\alpha(d)-\pi, \alpha(d)+\pi)$ if $d=b_{n}^{1}$. We recall that $\alpha(s)$ is the angle between the direction of the $O x_{1}$ axis and the tangent vector $\tau_{x}$ to $\Gamma^{1}$ at the point $x(s)$.

Hence, $\alpha(d)=\alpha\left(a_{n}^{1}+0\right)$ if $d=a_{n}^{1}$ and $\alpha(d)=\alpha\left(b_{n}^{1}-0\right)$ if $d=b_{n}^{1}$. along $\Gamma^{1}$.

Thus, the angle $\varphi$ varies continuously in the neighbourhood of the endpoint $x(d)$, cut

We will use the notation $\mu_{1}(s)=\mu(s)|s-d|^{1 / 2}=Q^{-1}(s) \mu_{*}(s)|s-d|^{1 / 2}$ and put $\mu_{1}(d)=\mu_{1}\left(a_{n}^{1}\right)=\mu_{1}\left(a_{n}^{1}+0\right)$ if $d=a_{n}^{1}$, and $\mu_{1}(d)=\mu_{1}\left(b_{n}^{1}\right)=\mu_{1}\left(b_{n}^{1}-0\right)$ if $d=b_{n}^{1}$.

Using the representation of the derivatives of harmonic potentials in terms of Cauchy type integrals (see [4]) and using the properties of these integrals near the endpoints of the integration line, presented in [15], we can prove the following assertion.

Theorem 5.1. Let $u(x)$ be a solution to the Problem $U$ ensured by Theorem 4.5. Let $x(d)$ be an arbitrary endpoint of the cracks $\Gamma^{1}$, that is, $x(d) \in X$ and $d=a_{n}^{1}$ or $d=b_{n}^{1}$ for some $n=1, \ldots, N_{1}$. Then the derivatives of the solution to the Problem $U$ in the neighbourhood of $x(d)$ have the following asymptotic behaviour.

$$
\begin{aligned}
& \text { If } d=a_{n}^{1} \text {, then } \\
& \qquad \begin{aligned}
\frac{\partial}{\partial x_{1}} u(x)= & \frac{\mu_{1}\left(a_{n}^{1}\right)}{2\left|x-x\left(a_{n}^{1}\right)\right|^{1 / 2}} \sin \left(\frac{\varphi+\alpha\left(a_{n}^{1}\right)}{2}\right) \\
& -\frac{v\left(a_{n}^{1}\right)}{2 \pi}\left(-\sin \alpha\left(a_{n}^{1}\right) \ln \left|x-x\left(a_{n}^{1}\right)\right|+\varphi \cos \alpha\left(a_{n}^{1}\right)\right)+O(1), \\
\frac{\partial}{\partial x_{2}} u(x)= & -\frac{\mu_{1}\left(a_{n}^{1}\right)}{2\left|x-x\left(a_{n}^{1}\right)\right|^{1 / 2}} \cos \left(\frac{\varphi+\alpha\left(a_{n}^{1}\right)}{2}\right) \\
& -\frac{v\left(a_{n}^{1}\right)}{2 \pi}\left(\cos \alpha\left(a_{n}^{1}\right) \ln \left|x-x\left(a_{n}^{1}\right)\right|+\varphi \sin \alpha\left(a_{n}^{1}\right)\right)+O(1) .
\end{aligned}
\end{aligned}
$$


If $d=b_{n}^{1}$, then

$$
\begin{aligned}
\frac{\partial}{\partial x_{1}} u(x)= & -\frac{\mu_{1}\left(b_{n}^{1}\right)}{2\left|x-x\left(b_{n}^{1}\right)\right|^{1 / 2}} \cos \left(\frac{\varphi+\alpha\left(b_{n}^{1}\right)}{2}\right) \\
& +\frac{v\left(b_{n}^{1}\right)}{2 \pi}\left(-\sin \alpha\left(b_{n}^{1}\right) \ln \left|x-x\left(b_{n}^{1}\right)\right|+\varphi \cos \alpha\left(b_{n}^{1}\right)\right)+O(1), \\
\frac{\partial}{\partial x_{2}} u(x)= & -\frac{\mu_{1}\left(b_{n}^{1}\right)}{2\left|x-x\left(b_{n}^{1}\right)\right|^{1 / 2}} \sin \left(\frac{\varphi+\alpha\left(b_{n}^{1}\right)}{2}\right) \\
& +\frac{v\left(b_{n}^{1}\right)}{2 \pi}\left(\cos \alpha\left(b_{n}^{1}\right) \ln \left|x-x\left(b_{n}^{1}\right)\right|+\varphi \sin \alpha\left(b_{n}^{1}\right)\right)+O(1) .
\end{aligned}
$$

By $O(1)$ one denotes functions which are continuous at the endpoint $x(d)$. Moreover, the functions denoted by $O(1)$ are continuous in the neighbourhood of the endpoint $x(d)$ cut along $\Gamma^{1}$ and are continuously extensible to $\left(\Gamma^{1}\right)^{+}$and to $\left(\Gamma^{1}\right)^{-}$from this neighbourhood.

The formulas of the theorem demonstrate the following curious fact. In the general case, the derivatives of the solution to the Problem $U$ near the endpoint $x(d)$ of cracks $\Gamma^{1}$ behave as

$$
O\left(|x-x(d)|^{-1 / 2}\right)+O\left(\ln \left(\frac{1}{|x-x(d)|}\right)\right) \text {. }
$$

However, if $\mu_{1}(d)=v(d)=0$, then $\nabla u(x)$ will be bounded and even continuous at the endpoint $x(d) \in X$.

\section{References}

[1] V. I. Smirnov, A Course of Higher Mathematics, vol. IV, Gostehizdat, Moscow-Leningrad, 1951, English translation Pergamon Press, Oxford, UK,1964.

[2] V. S. Vladimirov, Equations of Mathematical Physics, Nauka, Moscow, Russia, 1981, English translation Mir Publishers, Moscow, Russia, 1984.

[3] A. N. Tikhonov and A. A. Samarskii, Equations of Mathematical Physics, GITTL, Moscow, Russia, 1951, English translation Pergamon Press, Oxford, UK, 1963.

[4] P. A. Krutitskii, "Dirichlet problem for the Helmholtz equation outside cuts in a plane," Computational Mathematics and Mathematical Physics, vol. 34, pp. 1073-1090, 1994.

[5] P. A. Krutitskii, "On a uniquely solvable integral equation in a mixed Dirichlet-Neumann problem of acoustic scattering," Quarterly of Applied Mathematics, vol. 59, no. 3, pp. 493-506, 2001.

[6] P. A. Krutitskii, "Method of interior boundaries in a mixed problem of acoustic scattering," Mathematical Problems in Engineering, vol. 5, pp. 173-192, 1999.

[7] S. L. Sobolev, Some Applications of Functional Analysis to Mathematical Physics, Nauka, Moscow, Russia, 1988.

[8] I. K. Lifanov, Singular Integral Equations and Discrete Vortices, VSP, Zeist, The Netherlands, 1996.

[9] P. A. Krutitskii, D. Y. Kwak, and Y. K. Hyon, "Numerical treatment of a skew-derivative problem for the Laplace equation in the exterior of an open arc," Journal of Engineering Mathematics, vol. 59, no. 1, pp. 25-60, 2007.

[10] L. A. de Lacerda, L. C. Wrobel, H. Power, and W. J. Mansur, "A novel boundary integral formulation for three-dimensional analysis of thin acoustic barriers over an impedance plane," Journal of the Acoustical Society of America, vol. 104, no. 2, pp. 671-678, 1998. 
[11] J. António, A. Tadeu, and P. A. Mendes, "A 2.5D traction boundary element method formulation applied to the study of wave propagation in a fluid layer hosting a thin rigid body," Journal of Computational Acoustics, vol. 16, no. 2, pp. 177-198, 2008.

[12] A. Tadeu, J. António, L. Godinho, and P. Amado Mendes, "Simulation of sound absorption in 2D thin elements using a coupled BEM/TBEM formulation in the presence of fixed and moving 3D sources," Journal of Sound and Vibration, vol. 331, no. 10, pp. 2386-2403, 2012.

[13] D. I. Bardzokas, M. L. Filshtinsky, and L. A. Filshtinsky, Mathematical Methods in Electro-MagnetoElasticity, Springer, Berlin, Germany, 2010.

[14] P. A. Krutitskii and N. C. Krutitskaya, "The Dirichlet problem for equations of the static theory of elasticity outside curvilinear cracks on the plane," Differential Equations, vol. 45, no. 9, pp. 1285-1299, 2009.

[15] N. I. Muskhelishili, Singular Integral Equations, Nauka, Moscow, Russia, 1968, English translation Noordhoff, Groningen, The Netherlands, 1972.

[16] D. Gilbarg and N. S. Trudinger, Elliptic Partial Differential Equations of Second Order, Springer, Berlin, Germany, 1983, Russian translation Nauka, Moscow, Russia, 1989.

[17] V. A. Trénoguine, Functional Analysis, Nauka, Moscow, Russia, 1980, French translation Mir Publishers, Moscow, Russia, 1985.

[18] S. G. Krein, Ed., Functional Analysis, Nauka, Moscow, Russia, 1964, English translation WoltersNoordhoff Publishing, Groningen, The Netherlands, 1972.

[19] A. N. Kolmogorov and S. V. Fomin, Elements of Function Theory and Functional Analysis, Nauka, Moscow, Russia, 1981, English translation Dover, New York, NY, USA, 1999.

[20] L. V. Kantorovich and G. P. Akilov, Functional Analysis, Nauka, Moscow, Russia, 1977, English translation Pergamon Press, Oxford, UK, 1982. 


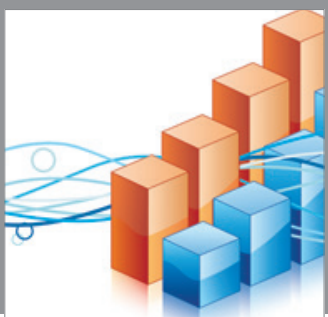

Advances in

Operations Research

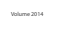

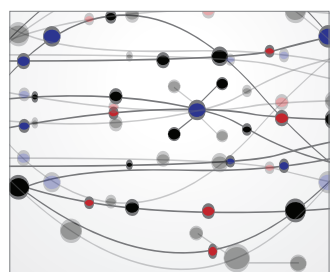

\section{The Scientific} World Journal
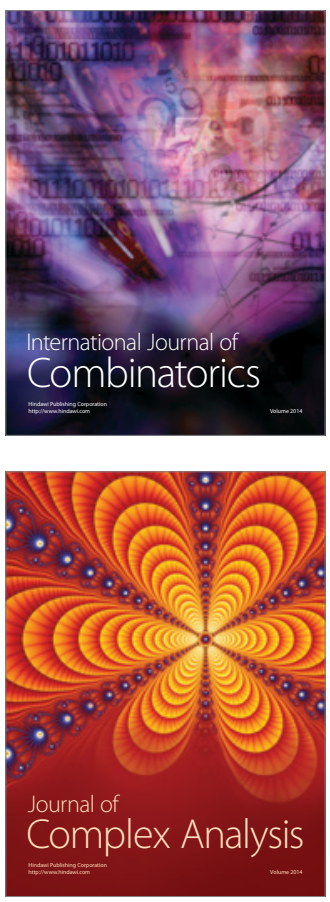

International Journal of

Mathematics and

Mathematical

Sciences
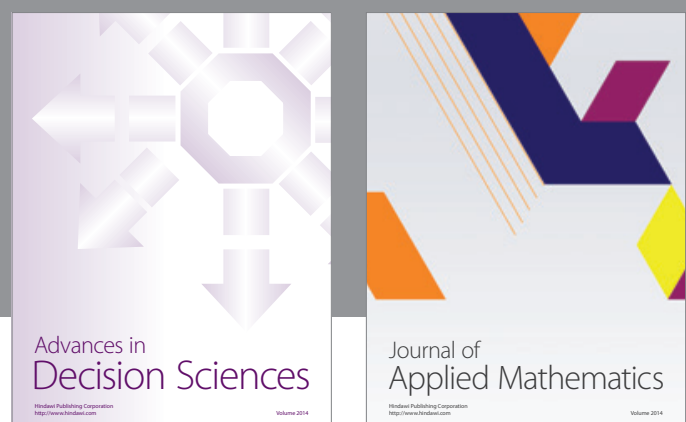

Journal of

Applied Mathematics
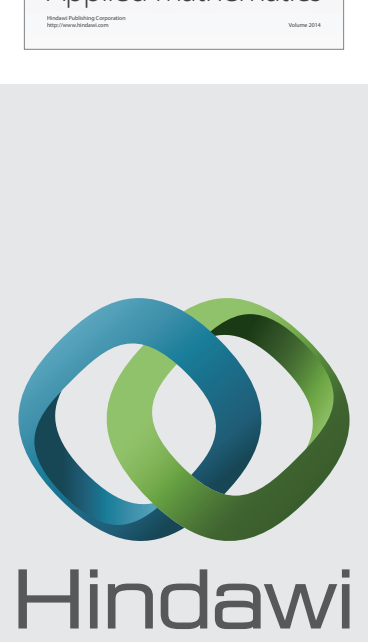

Submit your manuscripts at http://www.hindawi.com
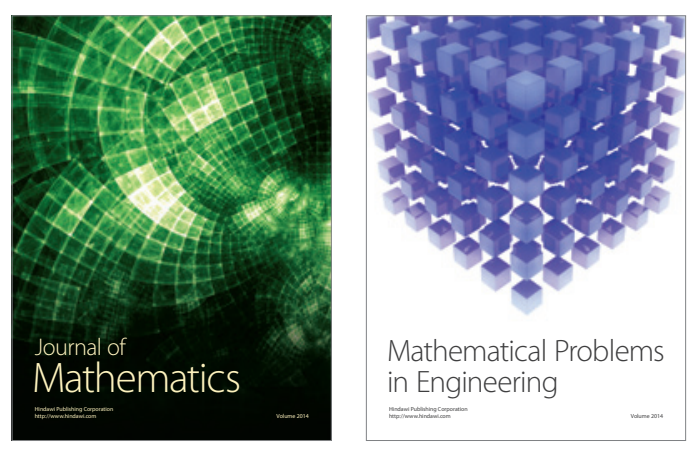

Mathematical Problems in Engineering
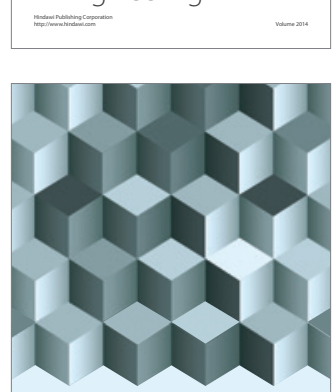

Journal of

Function Spaces
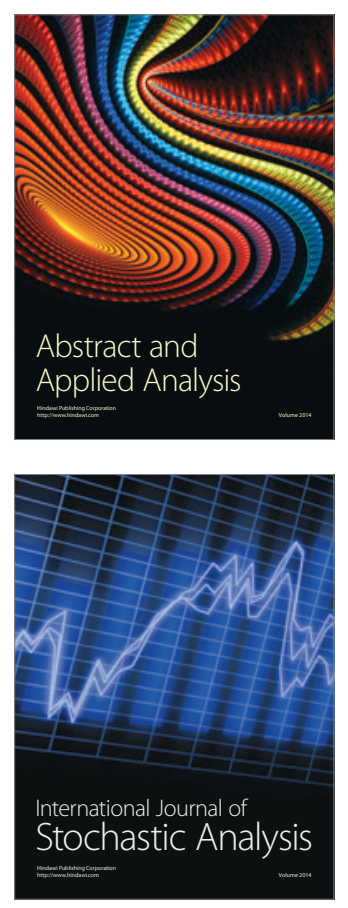

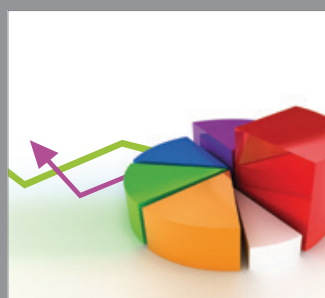

ournal of

Probability and Statistics

Promensencen
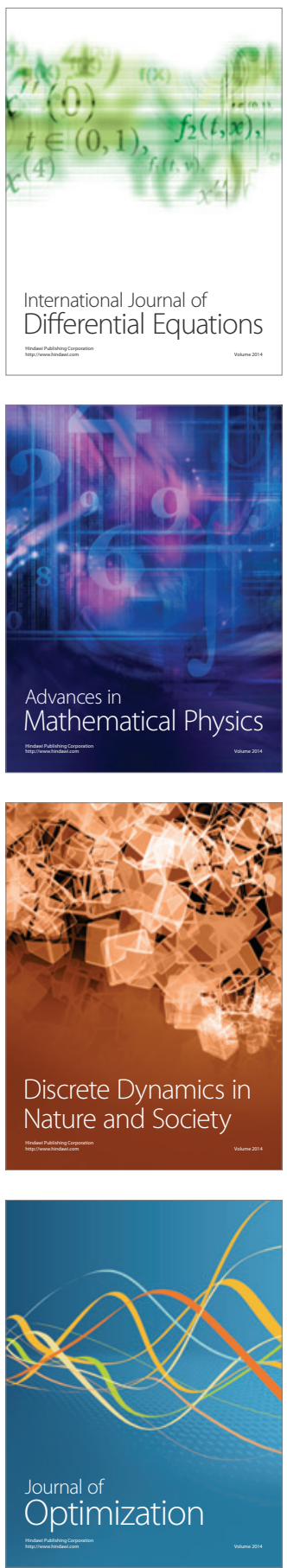